\title{
Selective impairment of methylation maintenance is the major cause of DNA methylation reprogramming in the early embryo
}

\author{
Julia Arand ${ }^{1,4}$, Mark Wossidlo ${ }^{1,5 \dagger}$, Konstantin Lepikhov ${ }^{1 \dagger}$, Julian R Peat ${ }^{2}$, Wolf Reik ${ }^{2,3}$ and Jörn Walter ${ }^{1 *}$
}

\begin{abstract}
Background: DNA methylomes are extensively reprogrammed during mouse pre-implantation and early germ cell development. The main feature of this reprogramming is a genome-wide decrease in 5-methylcytosine $(5 \mathrm{mC})$. Standard high-resolution single-stranded bisulfite sequencing techniques do not allow discrimination of the underlying passive (replication-dependent) or active enzymatic mechanisms of $5 \mathrm{mC}$ loss. We approached this problem by generating high-resolution deep hairpin bisulfite sequencing (DHBS) maps, allowing us to follow the patterns of symmetric DNA methylation at CpGs dyads on both DNA strands over single replications.

Results: We compared DHBS maps of repetitive elements in the developing zygote, the early embryo, and primordial germ cells (PGCs) at defined stages of development. In the zygote, we observed distinct effects in paternal and maternal chromosomes. A significant loss of paternal DNA methylation was linked to replication and to an increase in continuous and dispersed hemimethylated CpG dyad patterns. Overall methylation levels at maternal copies remained largely unchanged, but showed an increased level of dispersed hemi-methylated CpG dyads. After the first cell cycle, the combined DHBS patterns of paternal and maternal chromosomes remained unchanged over the next three cell divisions. By contrast, in PGCs the DNA demethylation process was continuous, as seen by a consistent decrease in fully methylated CpG dyads over consecutive cell divisions.

Conclusions: The main driver of DNA demethylation in germ cells and in the zygote is partial impairment of maintenance of symmetric DNA methylation at CpG dyads. In the embryo, this passive demethylation is restricted to the first cell division, whereas it continues over several cell divisions in germ cells. The dispersed patterns of CpG dyads in the early-cleavage embryo suggest a continuous partial (and to a low extent active) loss of methylation apparently compensated for by selective de novo methylation. We conclude that a combination of passive and active demethylation events counteracted by de novo methylation are involved in the distinct reprogramming dynamics of DNA methylomes in the zygote, the early embryo, and PGCs.
\end{abstract}

Keywords: DNA methylation reprogramming, Pre-implantation development, DNA methylation pattern, Deep hairpin bisulfite sequencing

\section{Background}

The life cycle of mammals is characterized by two phases of major epigenetic reprogramming: first during migration of the primordial germ cells (PGCs) to the genital ridge in the developing embryo, and the second after fertilization during pre-implantation development

\footnotetext{
* Correspondence: j.walter@mx.uni-saarland.de

${ }^{\dagger}$ Equal contributors

'University of Saarland, FR 8.3, Biological Sciences, Genetics/Epigenetics, Campus A2.4, 66123 Saarbrücken, Germany

Full list of author information is available at the end of the article
}

[1]. These phases of epigenetic reprogramming involve changes in histone modifications and the activation of pluripotency-associated factors. Most intriguing is the accompanying reprogramming of DNA methylation, mainly characterized by a genome-wide decrease in DNA methylation [2-9]. The molecular control mechanisms for both genome-wide DNA demethylation processes remain unclear. In principle, demethylation of 5 -methylcytosine $(5 \mathrm{mC})$ can be accomplished via an active enzymatic process or a passive replication-dependent process. Active DNA demethylation involves enzymes that 
remove either the methyl group or the whole base, accompanied by activation of ubiquitous DNA repair pathways [10].

In PGCs, a large proportion of DNA demethylation appears to occur by replication-associated passive demethylation, most likely influenced by 5-hydroxymethylcytosine $(5 \mathrm{hmC})[5,11,12]$. However, mechanisms of active DNA demethylation by enzymatic conversion of $5 \mathrm{mC}$ (or $5 \mathrm{hmC}$ ) are also likely to contribute. Thus, mechanisms involving the deamination of $5 \mathrm{mC}$ to thymine by activation-induced deaminase (AID) or other non-deamination dependent repair pathways have been suggested $[2,13]$.

In the zygote, a substantial loss of $5 \mathrm{mC}$ in the paternal pronucleus before replication has been shown by immunofluorescence (IF) analyses. For a long time, this was interpreted to represent genome-wide active loss of DNA methylation before replication [14-16]. An observed major drop in DNA methylation by bisulfite sequencing after replication [16] indicated that $5 \mathrm{mC}$ is not immediately replaced with unmodified cytosine but rather converted into a different chemical status. Indeed with the discovery of $5 \mathrm{hmC}$, it became clear that the conversion of $5 \mathrm{mC}$ into $5 \mathrm{hmC}$ and other oxidized forms catalyzed by Tet 3 are likely mechanisms to initiate the progressive loss of $5 \mathrm{mC}$ [17-19]. Hence, the idea of an active paternal genome demethylation had to be reconsidered. In addition, as $5 \mathrm{hmC}$ appears to be diluted during further cleavage stages, this modification is likely to be the major cause of DNA demethylation during the cleavage stages, caused by a continued impairment of maintenance methylation function of the DNA methyltransferase Dnmt1 [20]. However, even high-resolution IF analyses left open the question of how doublestranded DNA methylation patterns are affected in the first rounds of cell divisions. Recent studies using enrichment-based profiling (reduced representation bisulfite sequencing; RRBS) and genome-wide bisulfite sequencing provided evidence that the most dramatic effect of DNA demethylation takes place at the zygotic stage $[6,9]$. However, although these analyses revealed an overall dilution effect on DNA methylation, they did not address how DNA methylation patterns on both complementary DNA strands (complementary CpG dinucleotide; CpG dyads) are affected, and therefore could not draw conclusions on the possible mechanisms controlling DNA methylation.

In this study, we address these open questions by simultaneously analyzing the changes in DNA methylation patterns on both complementary DNA strands during the early phases of mouse development. We used hairpin bisulfite sequencing $[21,22]$ to investigate the replicationdependent DNA methylation pattern dynamics at specific repetitive elements such as the L1Md_Tf (hereafter referred to as L1), major satellites (mSat) and IAPLTR1
(IAP). L1 and mSat were chosen because they have previously been shown to undergo DNA demethylation, whereas IAPs were reported to be resistant to DNA demethylation in the zygote $[9,16,23,24]$. Our analysis follows the fate of DNA methylation at these elements, starting from mouse germ cells over the first cleavage stages up to the blastocyst stage and PGCs. In addition, zygotes and two-cell embryos were analyzed at precisely timed stages during the cell cycle in order to discriminate between pre-replicative and post-replicative states. Additionally, we separately isolated maternal and paternal zygotic pronuclei for our analyses in order to address differences previously reported for both sets of chromosomes $[6,14,25]$. Our comparative analysis provides clear evidence for the presence of a DNA methylation maintenance function in early embryos, which is partially impaired during the first cell cycle. We also found continuous presence of hemimethylated CpG dyads across the first cell divisions. Our findings suggest a complex interplay between possible mechanisms of DNA methylation reprogramming (that is, demethylation and de novo methylation) and DNA methylation maintenance in the early mouse embryo.

\section{Results}

DNA methylation reprogramming of L1, $\mathrm{mSat}$, and IAP in the zygote is characterized by an increasing amount of hemimethylated $\mathrm{CpG}$ dyads

To precisely determine DNA methylation symmetry of individual CpG dyads at single nucleotide resolution, we performed deep hairpin bisulfite sequencing (DHBS). We first determined the ground state of methylation at L1, IAP, and mSat in mature germ cells (sperm and oocytes). In line with previous data, we found that mSat are hypomethylated and IAP hypermethylated in both oocytes and sperm, whereas L1 elements are hypermethylated in sperm and hypomethylated in oocytes (Figure 1) $[9,23,24]$. It should be note that bisulfite sequencing cannot distinguish $5 \mathrm{mC}$ from $5 \mathrm{hmC}$ or unmodified $\mathrm{C}$ from 5-formylcytosine $(5 \mathrm{fC})$ or 5carboxycytosine $(5 \mathrm{caC})$, therefore the term "methylated DNA sequences" refers hereafter to the sum of $5 \mathrm{mC}$ and $5 \mathrm{hmC}$, and accordingly, hemimethylated CpG dyads are the sum of hemi- $5 \mathrm{mC}$ and hemi$5 \mathrm{hmC}$. DHBS showed that the methylated dyads of both sperm and oocyte chromosomes contain a high level of fully methylated CpG dyads (Figure 1). In early pre-replicative zygotes, DNA methylation represents the average of oocyte and sperm values, with the number of fully methylated $\mathrm{CpG}$ dyads initially remaining unaltered (Figure 1). At post-replicative pronuclear stages of the zygote (for examples of staging see Additional file 1), DNA methylation patterns change dramatically, with an extensive loss of fully methylated CpG dyads, accompanied by a 


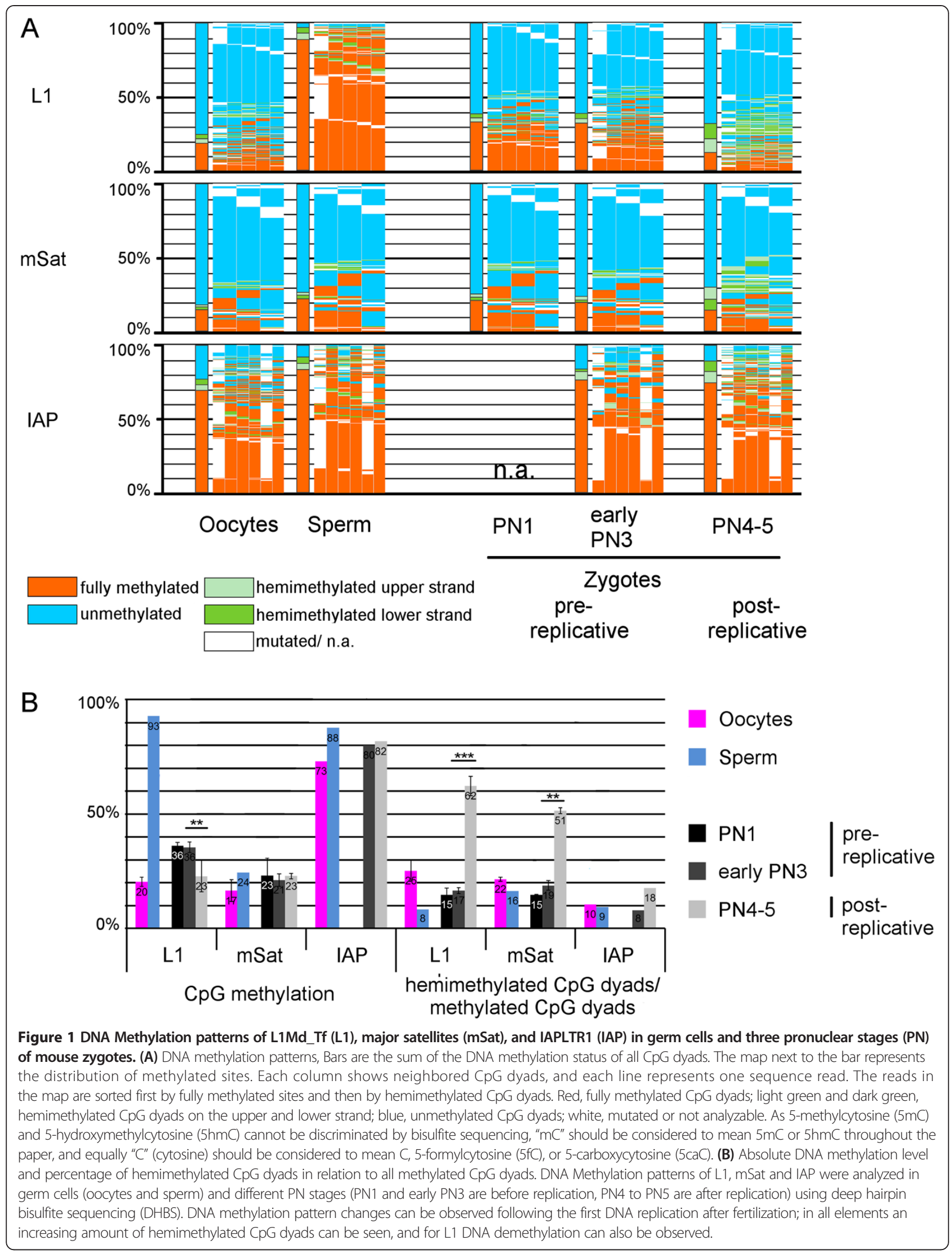


strong increase in hemimethylated CpG dyads. After replication, more than $50 \%$ of all methylated CpG dyads of L1 and $\mathrm{mSat}$ copies are in a hemimethylated state. By contrast, at IAP, almost all methylated CpG dyads remain fully methylated. Hence, despite an overall dramatic shift towards hemimethylated $\mathrm{CpG}$ dyads, a substantial proportion of fully methylated CpG dyads remain in certain L1 and mSat copies and in almost all IAP. This finding argues for a selective control of maintenance methylation during the first zygotic replication.

\section{DNA methylation patterns change differently in maternal and paternal pronuclei}

IF analyses of zygotes with antibodies against $5 \mathrm{mC}$ and $5 \mathrm{hmC}$ strongly suggested that the conversion of $5 \mathrm{mC}$ to $5 \mathrm{hmC}$ is particularly pronounced in the paternal DNA [17-19], and may serve as a signal for DNA demethylation. Using single nucleotide polymorphisms (SNPs) in single copy genes, we and others have found a more pronounced zygotic demethylation in paternal copies $[6,9,25]$. To investigate this on a broader genomic scale, we performed DHBS and conventional single-stranded bisulfite sequencing on DNA from isolated paternal and maternal pronuclei, and compared the methylation patterns to those of the corresponding germ cells (Figure 2; see Additional file 2). The analysis confirmed a strong and significant reduction in DNA methylation in paternal L1 elements, whereas the reduction in mSat was comparatively small (with low significance) (Figure 2). The demethylation of paternal L1 copies was associated with an increase in the ratio of hemimethylated CpG dyads (Figure 2b). Interestingly, the overall level of L1 and mSat methylation in maternal pronuclei remained largely unchanged, whereas the composition of DNA methylation patterns showed a strong increase in hemimethylated CpG dyads relative to all methylated dyads. We have recently shown that the amount of $5 \mathrm{hmC}$ also increases in the maternal pronuclei, whereas the level of $5 \mathrm{mC}$ decreases [19]. The increased levels of hemimethylated dyads could be linked to this effect.

\section{Major DNA methylation changes in the zygote require DNA synthesis and replication}

To better understand the connection between DNA methylation and DNA de novo synthesis, we analyzed zygotes treated with aphidicolin. Aphidicolin blocks all DNA synthesis but it does not affect zygotic pronuclear maturation (see Additional file 3). We found that DHBS methylation patterns of mSat copies in late-stage aphidicolin-treated zygotes remained unchanged compared with those of pre-replicative zygotes (Figure 3a,b). In L1 copies, we found a very small decrease in DNA methylation, visible as altered composition of hemimethylated and fully methylated sequences (Figure 3b). We conclude that the observed major changes in DNA methylation $(5 \mathrm{mC}$ and $5 \mathrm{hmC}$ ) in the zygote are mainly dependent on DNA synthesis (DNA repair and DNA replication).

Next, we investigated the effect of blocking all DNA methylation reactions (de novo and maintenance) during the first cell cycle, with the goal of detecting changes in DNA methylation patterns that are attributable to active DNA demethylation but are replication associated. To achieve this, we injected mRNA of the SAMase gene into early, pre-replicative zygotes. SAMase is a T3 bacteriophage-specific enzyme, which degrades Sadenosyl-methionine (SAM) [26]. The depletion of endogenous SAM pool by SAMase blocks all methylation reactions in which SAM serves as a methyl group donor. Expression of SAMase in zygotes led to visible reduction of $5 \mathrm{mC}$ when the resulting two-cell embryos were analyzed by IF (see Additional file 4). In paternal pronuclei of post-replicative zygotes, the perinucleolar rings, mainly enriched with $\mathrm{mSat}$ repeats, are usually positively stained by anti-5mC antibody, but when SAMase was expressed, the IF signals at perinucleolar rings were strongly reduced (see Additional file 4).

The DHBS methylation patterns obtained from the SAMase-treated post-replicative stages showed almost complete lack of fully methylated CpG dyads (Figure 3c), validating the inhibition of de novo and maintenance methylation. To identify events attributable to active DNA demethylation, we compared these DNA methylation patterns with those obtained by simulated replication without any maintenance or de novo methylation events of pre-replicative zygotes (in silico replication; see Methods). Neither the methylation patterns nor the levels showed significant differences (Figure 3c,d), indicating that mainly passive DNA demethylation events occur during the first replication in the zygote.

Taken together, both experiments clearly show that DNA synthesis and replication is necessary for substantial DNA demethylation. In addition, they suggest that partially impaired maintenance methylation is likely to be the major cause of $5 \mathrm{mC} / 5 \mathrm{hmC}$ demethylation in the paternal chromosomes during the first cell cycle.

We note that our data do not exclude a (minor) contribution by active DNA demethylation mechanisms. Two observations support a minor contribution of active DNA demethylation: First, we found that in aphidicolintreated zygotes, there was a small but recognizable change in DHBS patterns, when early zygotes were compared against late aphidicolin-treated zygotes, suggesting a small replication-independent change in the $5 \mathrm{mC}$ / $5 \mathrm{hmC}$ content (for example increase in hemimethylated CpG dyads in L1) (Figure 3), in line with our previous observations [16]. Second, the strong increase in unmethylated $\mathrm{CpG}$ positions in paternal pronuclei DNA cannot be explained by a selective passive dilution 
mechanism alone (Figure 2). The contribution of oxidized forms of $5 \mathrm{mC}(5 \mathrm{fC}, 5 \mathrm{caC})$ to the observed increase in "unmethylated" cytosines remains unclear because the standard bisulfite technology does not discriminate unmodified cytosine and $5 \mathrm{fC}$ or $5 \mathrm{caC}$. It is likely that some of the methylation changes are influenced by prereplicative and post-replicative oxidation of $5 \mathrm{hmC}$ into $5 \mathrm{fC}$ or $5 \mathrm{caC}$, which would not be detected by bisulfite sequencing. These findings indicate a minor contribution of active demethylation with the conversion of $5 \mathrm{mC}$ to unmodified cytosines.

Mosaic DNA methylation during the first cleavage stages suggests a constant loss and gain of methylation Having determined the baseline state of DNA methylation at the end of the first cell cycle, we then followed

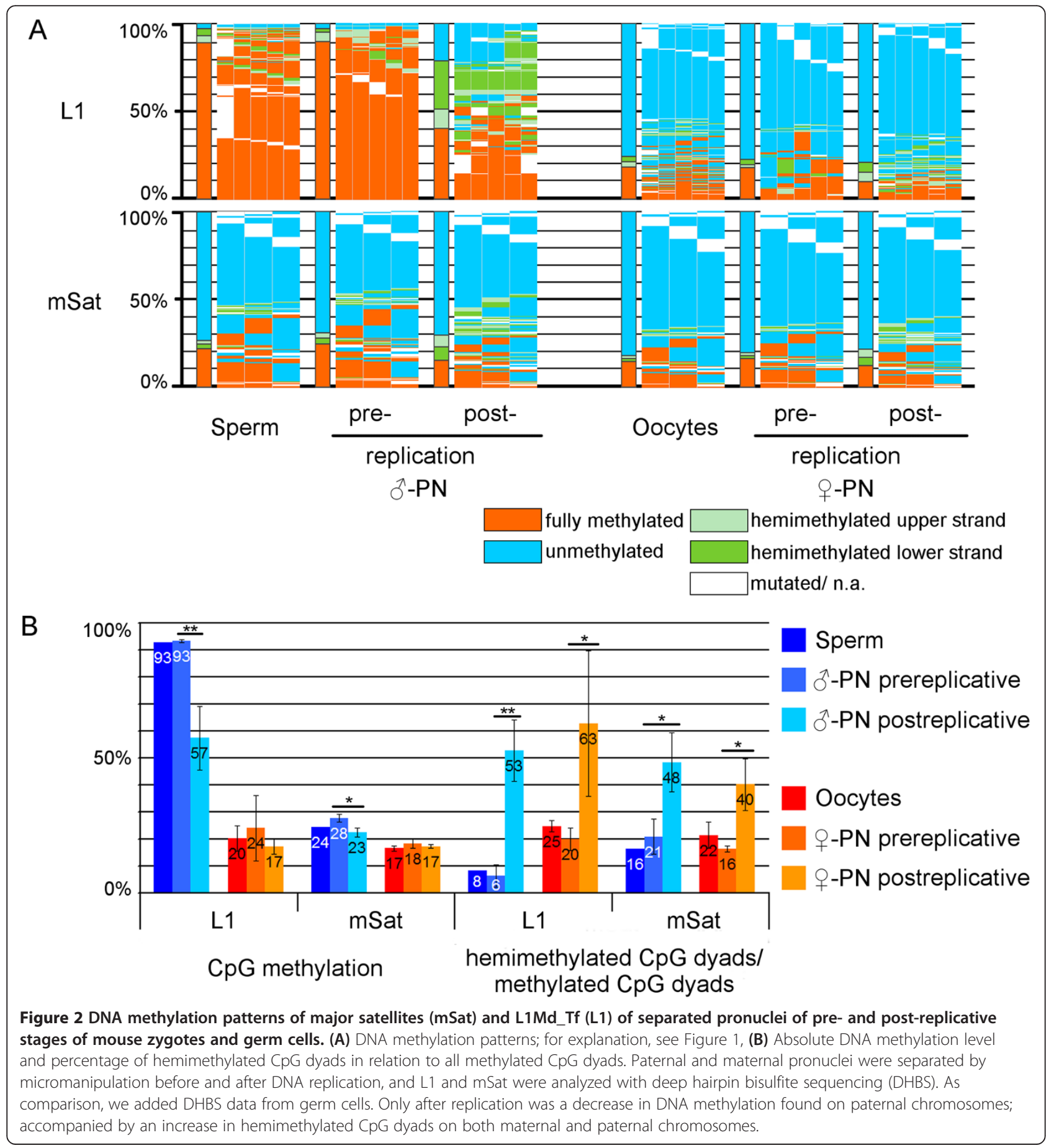




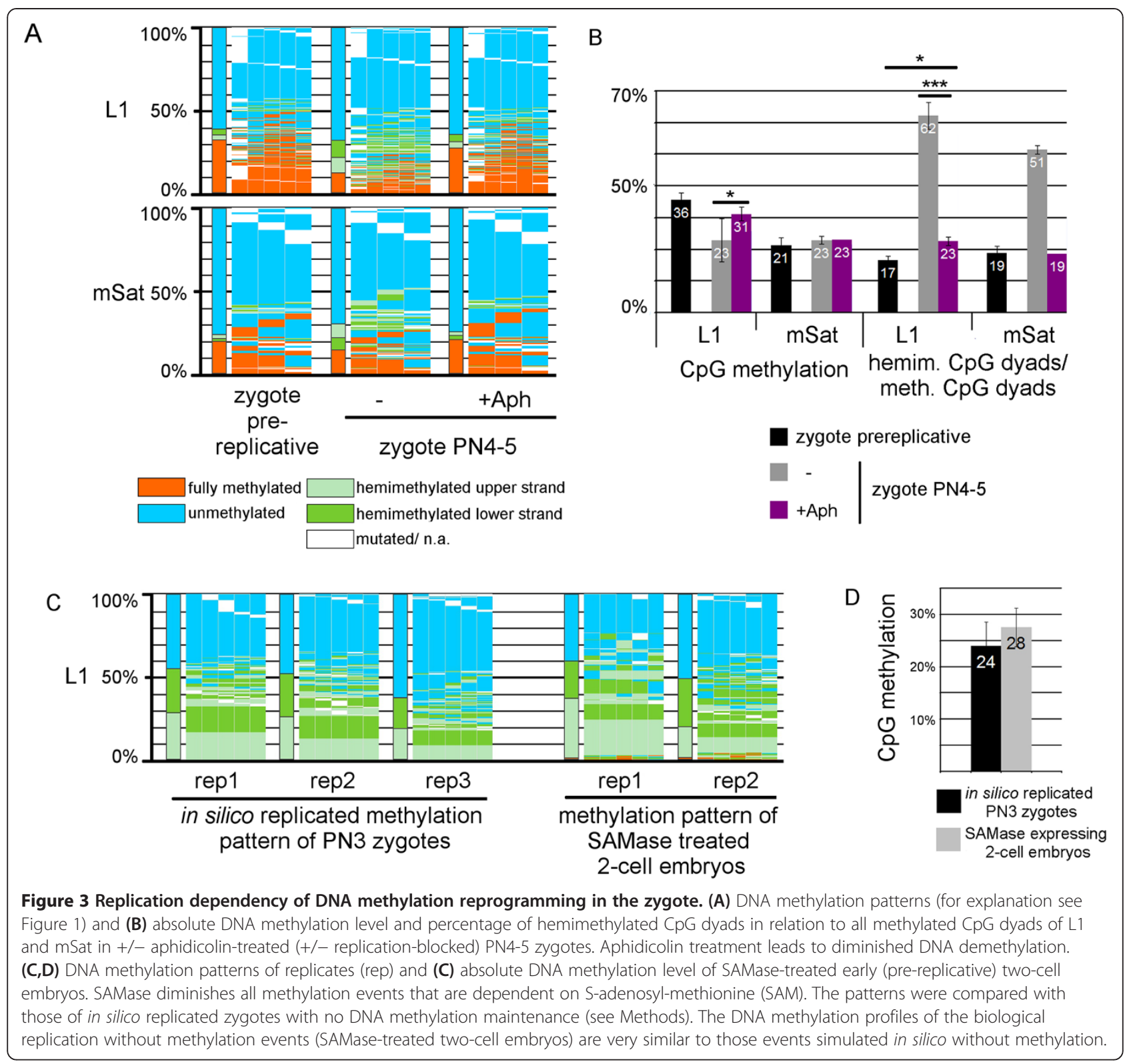

the DNA methylation patterns during the first cleavage stages. Previous work has suggested continuous passive DNA demethylation and/or non-maintenance of $5 \mathrm{hmC}[1,20]$, leading to further dilution of DNA methylation. However, when we followed the methylation pattern of two-cell embryos before and after replication, we did not observe a significant decrease in DNA methylation during the second DNA replication event (Figure 4). In fact, in IAP elements we actually found an increase in fully methylated $\mathrm{CpG}$ dyads at this point.

Next, we analyzed the DNA methylation in embryos collected at 12-hour intervals (Figure 4, Additional file 5). Until the early morula ( 8 to 16 -cell stage, 2.5 days post-coitum $(\mathrm{dpc})$ ) the overall methylation level and proportional distribution of all elements remained largely constant, accompanied by consistently high proportions of hemimethylated $\mathrm{CpG}$ dyads relative to all methylated $\mathrm{CpG}$ dyads. Only at the transition between late morula (16 to 32 cells, $3 \mathrm{dpc}$ ) and blastocyst stage ( $>64$ cells, $3.5 \mathrm{dpc}$ ), we observed a further decrease in DNA methylation at L1 and IAP elements, along with an increase in hemimethylated CpG dyads (Figure 4).

The maintenance of a high proportion of dispersed hemimethylated positions in maternal sequences in the zygote and between the two-cell and morula stages suggests that methylation maintenance involves a "balanced" loss and gain of methylation over several rounds of replication. A persistence of dispersed methylation profiles at $\mathrm{CPG}$ dyads can be caused by 


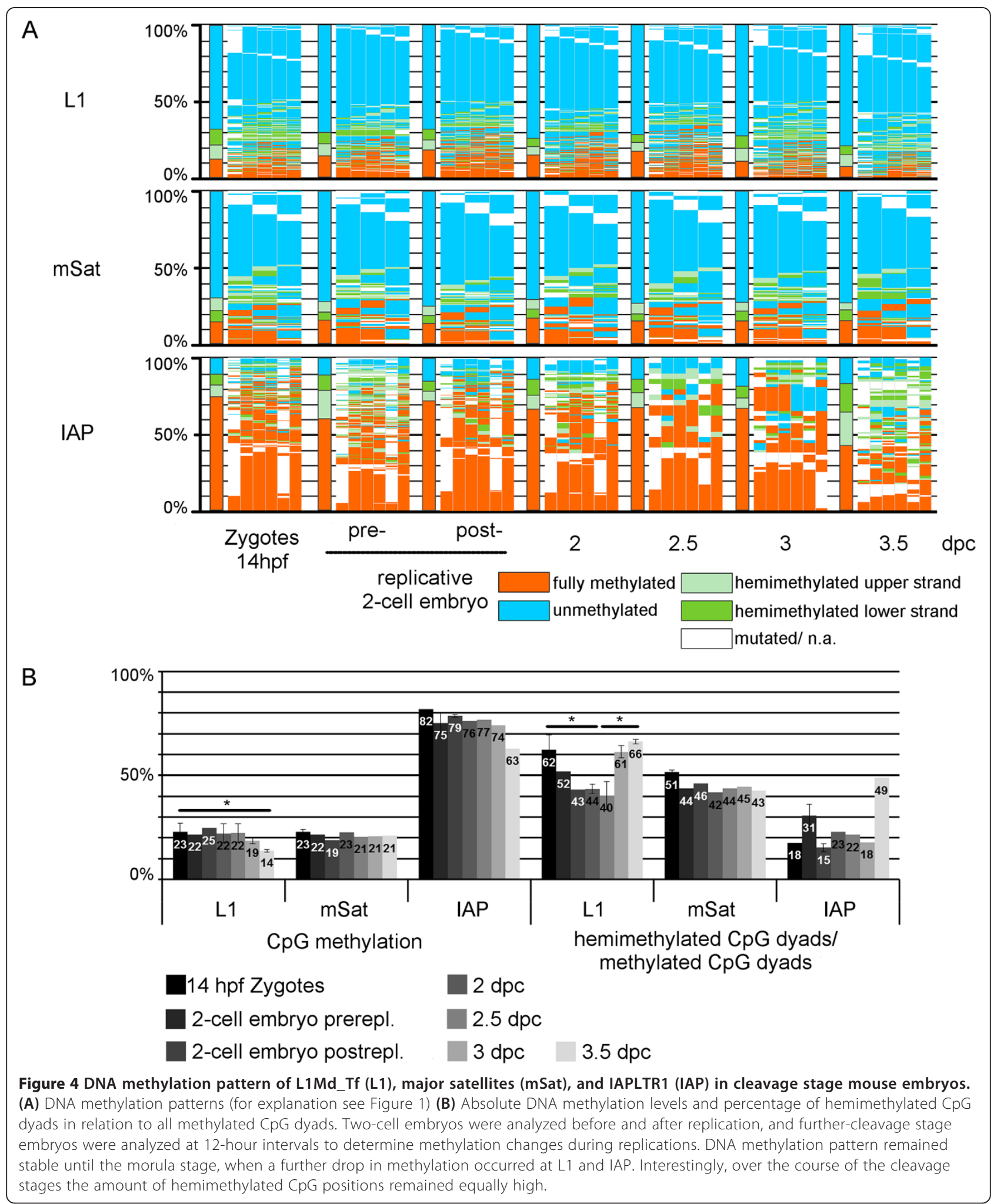

constantly maintaining high levels of highly oxidized forms (not detectable by bisulfite sequencing) and/or by a loss (impaired maintenance) and re-gain of $5 \mathrm{mC}$ by de novo methylation. As Tet3 is known to be absent from the two-cell stage onwards, the constant de novo methylation scenario is more likely to occur. 
DNA is progressively demethylated in replicating PGCs

Low coverage and genome-wide data suggested a stepwise decrease in DNA methylation during PGC development $[4,5,27,28]$. To systematically investigate if this stepwise loss of DNA demethylation in PGCs is linked to replication-dependent accumulation of hemimethylated CpG dyads, we performed DHBS for mSat, IAP, and L1 in staged PGCs and somatic cells between 9.5 $\mathrm{dpc}$ and $13.5 \mathrm{dpc}$ (see also [5] for L1). In early PGCs (9.5 dpc) DNA methylation was already reduced compared with surrounding somatic cells (Figure 5). Somatic cells showed a very low level of hemimethylated $\mathrm{CpG}$ dyads, whereas $50 \%$ of all methylated CpG dyads in $9.5 \mathrm{dpc}$ PGCs exhibited a hemimethylated state, indicating that the DNA demethylation process is already in progress in $9.5 \mathrm{dpc}$ PGCs. Between 9.5 and $13.5 \mathrm{dpc}$, we observed continuous progression of DNA demethylation, accompanied by a persistently high proportion of hemimethylated $\mathrm{CpG}$ dyads.

Hence, as in early embryos, we found a strong correlation between overall loss of DNA methylation and the presence of hemimethylated CpG dyads. This strongly argues for a continuous selective impairment of maintenance methylation as a major mechanism of demethylation in PGCs (see also [5]). In contrast to early-cleavage embryos, this process appears to occur continuously in PGCs over several replication cycles, and is apparently not accompanied by de novo methylation.

\section{Discussion}

In our study, we analyzed the fate of symmetrical DNA methylation across the first cell divisions in the mouse pre-implantation embryo and in PGC development. These developmental periods are characterized by an extensive reprogramming of genome-wide DNA methylation patterns, mainly extensive erasure of $5 \mathrm{mC}$. We used DHBS to precisely follow the dynamics of DNA methylation patterns in single DNA strands of cells isolated at defined stages of these reprogramming phases. This staged DHBS profiling allowed us to draw mechanistic interpretations from the fate of methylation on single DNA molecules. The analyzed repetitive elements represent widely dispersed different reprogramming classes resistant or sensitive to demethylation, which, as we recently reported, also recapitulated pattern formation at single gene loci in embryonic stem cells (ESCs) [21].

One major observation of our study is that DNA demethylation is mainly caused by partial impairment of DNA methylation maintenance during replication. This can be deduced from the significant increase in hemimethylated $\mathrm{CpG}$ dyads in both pre-implantation embryos and during PGC development. The second observation is that this process appears to be continuous during PGC development, but discontinuous in the developing early embryo. In the early embryo, a decrease in methylation occurred at two developmental points: in the zygote (mainly reducing the level of paternal methylation) and around $3 \mathrm{dpc}$ (that is, around the 32-cell stage). Between the two-cell stage and day 3 of development (mainly up to the 16-cell stage) the chromosomes maintained a largely constant level of methylation. At L1 and mSat elements, we observed the presence of dispersed hemimethylated CpG dyads. Such "noisy" patterns were maintained up to day 3 of embryonic development at a constant level (Figure 6). Similar noisy patterns are found in ESCs lacking Dnmt1 [21], or in ESCs cultured in 2i medium [29]. Shipony et al. also recently reported "noisy" CpG methylation for certain DNA regions of ES cell clones [30].

During the revision of our manuscript, two other groups published RRBS studies showing that passive demethylation is the main cause of DNA demethylation in the zygote [31,32]. Both groups also reported small but significant demethylation of maternal chromosomes in the zygote; however, their analysis was not sufficiently deep to detect that the demethylation of L1 and mSat sequences is counteracted and "balanced" by de novo methylation. They also did not follow this across subsequent cell divisions where we found this process to be maintained. We therefore postulate that de novo methylation (most likely by Dnmt3a) accompanies the reprogramming events in the early embryo as previously suggested [25]. In addition, the persistence of symmetrically methylated $\mathrm{CpG}$ dyads in IAP elements during the early-cleavage stages strongly suggests that the machinery for strict methylation maintenance must be present in the early embryo. In conclusion, our analysis provides a differentiated picture of the various mechanisms involved in shaping of a specific DNA methylation program following fertilization. We speculate the persistence of "noisy" patterns may be important for developmental potency and lineage decisions in the early embryo.

The molecular mechanisms responsible for selective impairment of maintenance methylation during the first cell cycle are still unclear. The conversion of $5 \mathrm{mC}$ to $5 \mathrm{hmC}, 5 \mathrm{fC}$, and $5 \mathrm{caC}$ may play a crucial role. Reprogramming of DNA methylation in the zygote can be initiated by the oxidation of $5 \mathrm{mC}$ by Tet3 $[17,19]$. Indeed, DNA demethylation of L1 was shown to be dependent on Tet3 activity [17], and in vitro data suggest that Dnmt1 fails to maintain methylation at CpG containing hydroxymethylated cytosines [33]. Furthermore, RRBS analysis of Tet3 KO zygotes suggests that replicationdependent demethylation is partly dependent on oxidation of $5 \mathrm{mC}$ by Tet3 [31,32]. The targeted avoidance of passive DNA demethylation can accordingly be executed via interaction with specific factors, such as Stella, which impairs oxidation by Tet3 [19,34]. Previous findings 
corroborate this assumption: the accumulation of hemimethylated $\mathrm{CpG}$ dyads in both pronuclei corresponds well with increase in $5 \mathrm{hmC}$, as $5 \mathrm{hmC}$ is also detected in maternal pronuclei at later stages $[9,19]$. Furthermore, another study published during the proofs of this manuscript suggests that there might be Tet3-dependent and other mechanisms-dependent demethylation pathways which are redundant [35].

In addition to a clear predominance of apparently passive demethylation mechanisms in the mouse zygote,

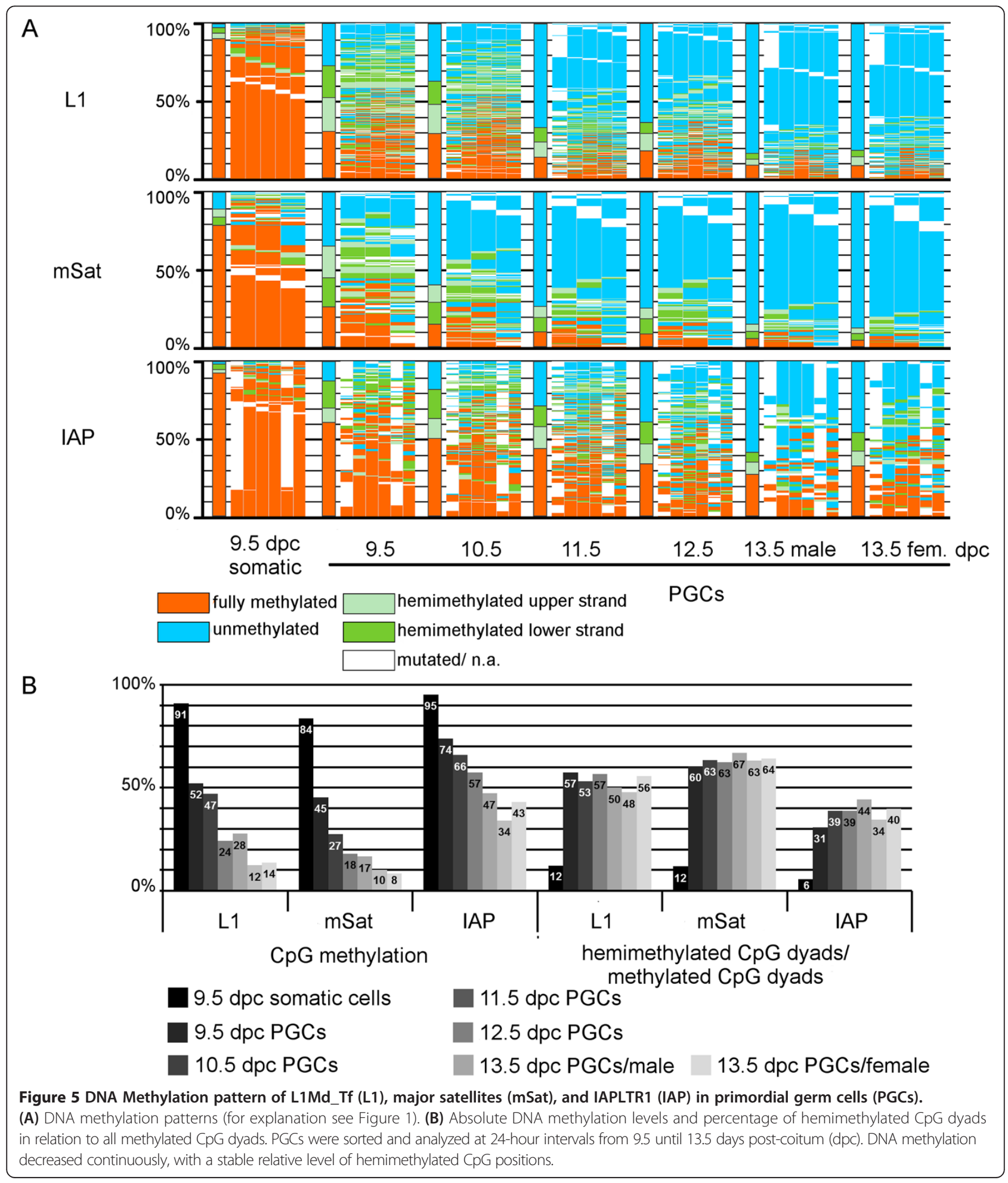




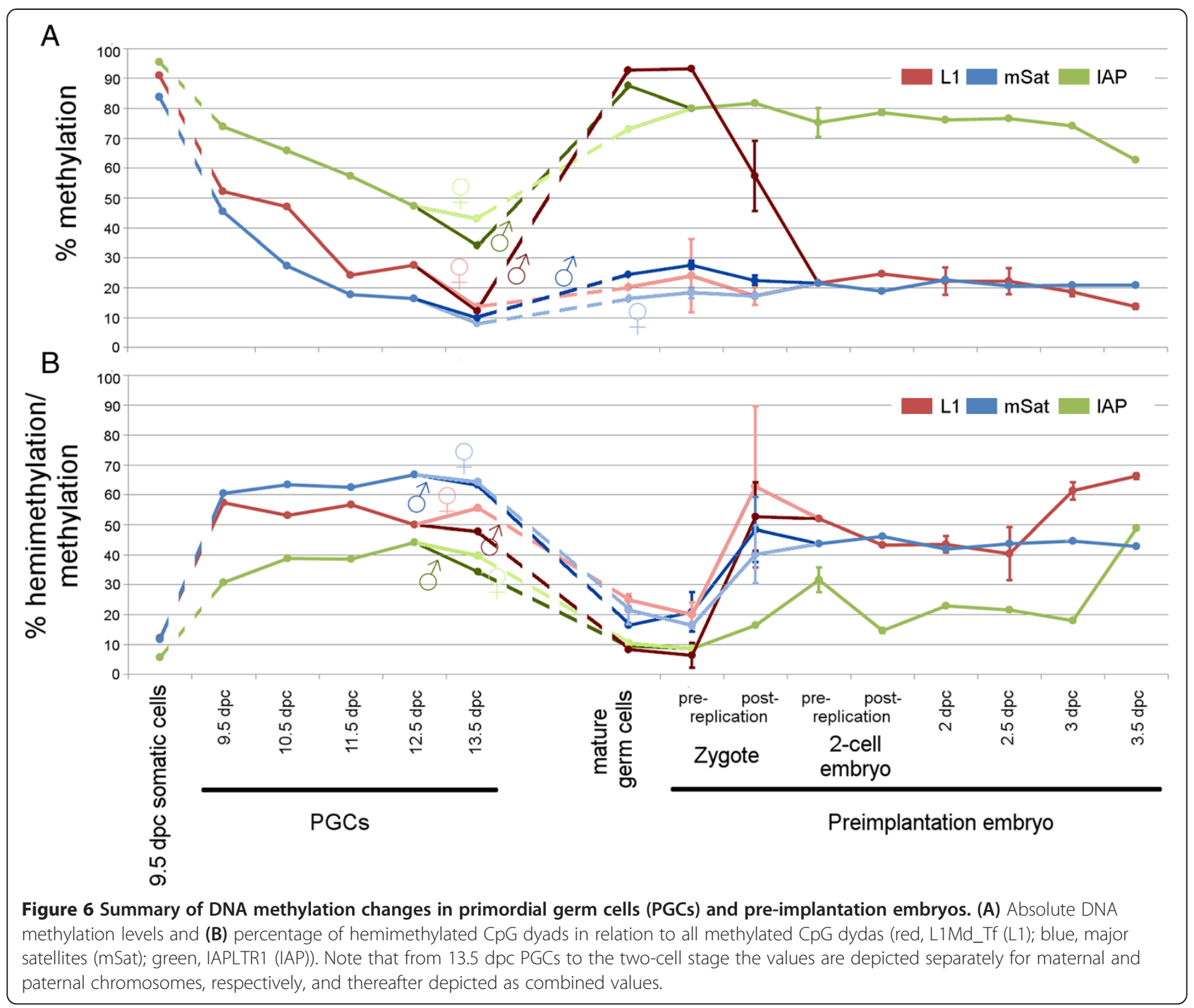

careful inspection of methylation patterns identified a lower prevalence of active DNA demethylation, in line with previous and two very recent reports $[16,24,25,31,32]$. Hence, a moderate and sequence-specific contribution of active mechanisms to DNA demethylation is apparently contributing to reprogramming. However, because the bisulfite reaction does not discriminate between unmodified cytosine, $5 \mathrm{fC}$, and $5 \mathrm{caC}[36,37]$, it is unclear whether our data indicate formation of unmodified cytosines by an active, probably repair-coupled, process, or if the changes in patterns simply reflect the generation of higher oxidized forms of $5 \mathrm{mC}$. Indeed, reports indicate the presence of $5 \mathrm{fC} / 5 \mathrm{caC}$ in the zygote using IF analysis [38] and a specifically modified bisulfite sequencing protocol [9]. However, a recent study found no or only very little detectable $5 \mathrm{fC} / 5 \mathrm{caC}$ at specific actively demethylated sequences in zygotes, and suggested that they are further processed by pathways such as base excision repair to yield unmodified cytosine [31].
High-resolution IF analysis in a previous study suggested a replication-dependent dilution of $5 \mathrm{hmC}$ during further cleavage stages [20]. However, this scenario does not correspond to our molecular findings beyond the first cleavage stage. The model of a cell division (replication)-dependent dilution of modified cytosines ( $5 \mathrm{hmC}$ or $5 \mathrm{mC}$ ) would predict a further decrease of bisulfite treatment-resistant cytosines. From the two-cell embryo stage up to the early morula stage, the overall methylation patterns remained remarkably stable, maintaining a constant amount of hemimethylated CpG dyads (Figure 4). The finding that a great proportion of $\mathrm{CpG}$ dyads retained a fully methylated state after continuous replication cycles indicates that maintenance methylation is not completely absent during the first cleavage stages, but that it is apparently impaired at selected sequences. These findings can be explained by the following scenario. The impairment of maintenance methylation by $5 \mathrm{hmC}$ is highest during the first cell cycle at selected sequences. In the absence of 
Tet3 and other factors at later stages [19], DNA methylation maintenance is impaired to a lesser extent and/or further passive loss is counteracted by enhanced de novo methylation carried out by de novo methyltransferases, probably by Dnmt3a, which has been shown to be present in the zygote and later stages [17]. By contrast, Dnmt3a and Dnmt3b are not expressed in PGCs ([39] and own unpublished observations), where DNA methylation continuously decreases during subsequent cell divisions (Figure 5).

This work underlines the need to more closely characterize the various contributions of DNA methyltransferase for DNA methylation persistence and their temporal control during early embryogenesis, in order to gain a better understanding of DNA methylation reprogramming processes.

\section{Conclusions}

Using DHBS, we have generated the first deep resolution methylation maps of $\mathrm{CpG}$ dyads of specific repetitive element classes across individual DNA replications and cell divisions in the early mouse embryo and during PGC formation (summarized in Figure 6). In PGCs, CpG methylation continuously decreases across consecutive cell divisions. This process is clearly linked to an accumulation of hemimethylated CpG dyads, reflecting a replication-dependent "passive" demethylation process. In the early embryo, such a process is confined to the paternal chromosomes, and occurs only during the first zygotic DNA replication. In the following cleavages and on maternal chromosomes in the late zygote, there is no loss of methylation but rather the maintenance of a constant degree of hemimethylated $\mathrm{CpG}$ dyad patterns at various repetitive elements. Our data suggest that in the embryo, incomplete passive and to a much lesser extent active demethylation mechanisms are antagonized by partial (de novo) methylation mechanisms to precisely maintain a development specific level of DNA methylation. Oxidation of $5 \mathrm{mC}$ by Tet enzymes is probably involved in the balance of these antagonistic enzymatic activities. In conclusion, during both major reprogramming phases in development, there is a rather dynamic DNA methylation landscape instead of a simple copying mechanism of the methylation pattern as seen in somatic cells. The establishment of these highly dynamic DNA methylation patterns is likely to be an important step in the generation of a totipotent and pluripotent epigenome and subsequent cell fate decisions in early embryogenesis.

\section{Methods}

All animal experiments were carried out according to German Animal Welfare law in agreement with the authorizing committee.

\section{In vitro fertilization of mouse oocytes and manipulation of zygotic development}

For in vitro fertilization (IVF), sperm was isolated from the cauda epididymis of adult (C57BL/ $6 \times$ CBA) F1 male mice, and pre-incubated for $1.5 \mathrm{~h}$ in modified Embryomax KSOM Embryo culture medium (Merck Millipore, Darmstadt, Germany) (3 $\mathrm{mg} \mathrm{BSA} / \mathrm{ml}$ and $5.56 \mathrm{mM}$ glucose in KSOM) supplemented with $27 \mathrm{mg} \mathrm{BSA} / \mathrm{ml}$. Mature oocytes from superovulated (C57BL/6 $\times$ CBA) F1 female mice were collected $14 \mathrm{~h}$ post-human chorionic gonadropin (hCG) injection according to the standard procedures [40]. Cumulus-oocyte complexes and capacitated sperm were placed into a $400 \mu \mathrm{l}$ drop of modified KSOM medium (see above) at $37^{\circ} \mathrm{C}$ in a humidified atmosphere of $5 \% \mathrm{CO}_{2}$ and $95 \%$ air. For the treatment with aphidicolin, $3 \mu \mathrm{g} / \mathrm{ml}$ aphidicolin was added at 4 hours post-fertilization (hpf). For collection of different PN stages, IVF-derived zygotes were stained with $5 \mu \mathrm{g} / \mathrm{ml}$ Hoechst 33342 for $30 \mathrm{~min}$ before the desired time points and correct PN staging, and contamination with sperm or cumulus cells was monitored by Hoechst staining and embryo by embryo selection under a fluorescent microscope. The classification of PN stages was performed as described previously $[15,16,41]$, with the pronuclear morphology and hpf taken into consideration.

\section{Collecting embryos from natural breeding}

Superovulated (C57BL/6 $\times$ CBA) F1 female mice were mated with $(\mathrm{C} 57 \mathrm{BL} / 6 \times \mathrm{CBA}) \mathrm{F} 1$ male mice. At embryonic day (E)1.5, two-cell embryos were flushed from the oviduct and incubated further in M16 (Sigma-Aldrich, St Louis, MO, USA). Embryos were collected at 12-hour intervals starting from $2 \mathrm{dpc}$ ( $2 \mathrm{dpc}$ : late 4-cell stage/ early 8 -cell stage; $2.5 \mathrm{dpc}$ : late 8 -cell stage/16 cell stage; 3 dpc: morula stage) until blastocyst stage at $3.5 \mathrm{dpc}$ (see Additional file 5).

\section{Pronuclei isolation}

IVF-derived zygotes at 7 or $13.5 \mathrm{hpf}$ were incubated with $5 \mu \mathrm{g} / \mathrm{ml}$ cytochalasin $\mathrm{B}, 2 \mu \mathrm{g} / \mathrm{ml}$ nocodazole, and $5 \mu \mathrm{g} / \mathrm{ml}$ Hoechst 33342 for $30 \mathrm{~min}$ in KSOM. Following this, the maternal and paternal pronuclei were separated using a micromanipulator under a Zeiss AxioVert $200 \mathrm{M}$ inverted microscope (Zeiss, Germany) in M2 medium without BSA supplemented with 1\% Polyvinylpyrrolidone (PVP), $5 \mu \mathrm{g} / \mathrm{ml}$ cytochalasin $\mathrm{B}$, and $2 \mu \mathrm{g} / \mathrm{ml}$ nocodazole. The parental origin of the pronuclei was determined by the size of the pronuclei and their location in relation to the polar body using Hoechst 33342 staining. Only clearly classifiable pronuclei were collected. 


\section{SAMase expression and injection into zygotes}

The T3 bacteriophage SAMase coding sequence was amplified by PCR from T3 bacteriophage DNA, and inserted into a pET28b0-based vector, containing an enhanced green fluorescent protein (eGFP) coding sequence, followed by the $3^{\prime}$ untranslated region sequence of the mouse TRF2 gene and downstream poly (83A) sequence (adopted from the pcDNA3.1EGFPpoly(A) plasmid, described in [42]). The resulting plasmid was used as template for in vitro transcription (MessageMax T7 ARCA-Capped Message transcription kit, Epicentre Biotechnologies, Madison, WI, USA) to produce mRNA, encoding for the SAMase-eGFP fusion protein. The mRNA was injected into early zygotes 2 to 4 hpf, and the injected zygotes were allowed to develop further for 16 hours until they reach late zygote or early two-cell stage (after first S-phase, before second $\mathrm{S}$-phase). The translation efficiency was monitored by eGFP fluorescence.

\section{Isolation of PGCs}

Genital ridges from Oct4-GFP transgenic embryos [43] were isolated from 9.5-13.5 dpc embryos then treated with trypsin, and single GFP-positive cells were collected manually using an inverted fluorescence microscope Zeiss AxioVert $200 \mathrm{M}$ and micromanipulators (TransferMan NK2; Eppendorf, Germany). The sex of the embryos at $13.5 \mathrm{dpc}$ was determined by the arrangement of the PGCs in the gonad. Each sample contained at least 40 PGCs. As a control, we collected GFP-negative cells from $9.5 \mathrm{dpc}$ embryos.

\section{Hairpin bisulfite analysis}

Embryos/pronuclei and a medium control from the last washing step were supplemented with $100 \mathrm{ng}$ salmon sperm DNA and treated with proteinase $\mathrm{K}(0.2 \mathrm{mg} / \mathrm{ml}$ in $2 \mathrm{mM}$ Tris- $\mathrm{HCl}, 1 \mathrm{mM}$ EDTA), followed by hairpin bisulfite analysis as described previously [21] with the following changes. We analyzed 5 to 15 embryos/pronuclei and 40 to 50 PGCs per biological replica. For L1 analysis, the restriction enzyme BsaWI was used ( 3 hours at $60^{\circ} \mathrm{C}$ ) and for IAP analysis, the following primers and PCR conditions were used: forward TTTTTTTTTTAG GAGAGTTATATTT, reverse ATCACTCCCTAATTAA CTACAAC, 45 cycles $\left(95^{\circ} \mathrm{C}\right.$ for 1 minute, $51^{\circ} \mathrm{C}$ for 1.5 minutes, $72^{\circ} \mathrm{C}$ for 1 minute). For $\mathrm{L} 1$ and $\mathrm{mSat}$, the cycle number for the PCR was increased to 45 for L1 and 40 for mSat, respectively. Details of the results of the hairpin bisulfite sequencing of the different biological replicates and the number of replicates analyzed are given (see Additional file 6). Raw data can be obtained upon request.

\section{In silico replication}

To mimic the situation of complete absence of DNA methylation maintenance (passive demethylation) during the first DNA replication in the zygote, we halved the methylation at all $\mathrm{CpG}$ dyads (pre-replicative state), while maintaining their relative neighborhood localization. Thus, unmethylated CpG dyads will give rise to two sequences with each having a completely unmethylated CpG dyad, hemimethylated $\mathrm{CpG}$ dyads will give rise to one sequence with a hemimethylated CpG dyad and the other with an unmethylated CpG dyad, and fully methylated CpG dyads will give rise to two sequences with hemimethylated CpG dyads.

\section{Additional files}

\begin{abstract}
Additional file 1: Representative images of Hoechst 33342-stained mouse zygotes. Discrimination of developed zygotes was performed by hours and the morphology of the pronuclei (PN) as described previously [16]. PN1 and early PN3 represent pre-replicative PN stages and PN4 to PN5 the post-replicative PN stages. PB, polar body.
\end{abstract}

Additional file 2: Comparison of DNA methylation level of L1_Md_Tf (L1) obtained by deep hairpin bisulfite sequencing (DHBS) and deep single strand bisulfite sequencing (DSSBS). DNA methylation analysis of L1 in germ cells and maternal and paternal pronuclei at different timepoints of the developing zygote with DHBS showed the same overall methylation level as the methylation of L1 with DSSBS.

Additional file 3: 5-ethynyl-2'-deoxyuridine (EdU) incorporation and phosphorylated Histone variant H2A.X ( $\gamma \mathrm{H} 2 \mathrm{~A} . \mathrm{X})$ staining of zygotes inhibited with aphidicolin. Aphidicolin-treated zygotes (4 to $14 \mathrm{~h}$ ) did not show any incorporation of nucleotides but still showed expansion of the pronuclei. PB, polar body.

Additional file 4: The influence of SAMase expression in zygotes on 5mC. (A) $5 \mathrm{mC}$ immunofluorescence (IF) staining in control and SAMase expressing 14-hour in vitro fertilization (IVF) post-replicative zygotes. (B) $5 \mathrm{mC}$ IF staining in control and SAMase expressing two-cell embryos. PB, polar body.

Additional file 5: Representative pictures of cleavage stage embryos used for hairpin bisulfite analysis of L1Md_Tf (L1), major satellites (mSat) and IAPLTR1 (IAP) from day 2 post-fertilisation (2 dpc, days post-coitum: late 4-cell to early 8-cell stage), $2.5 \mathrm{dpc}$ (early morula: 16 cell stage), $2.5 \mathrm{dpc}$ (late morula stage) to $3.5 \mathrm{dpc}$ (blastocyst stage).

Additional file 6: List of all analyzed samples with number of reads and DNA methylation states. Samples analyzed with Hairpin bisulfite sequencing with number of reads (\#reads), number of analyzed CpGs (\#CpGs), conversion rate of the hairpin linker (conversion), and ratio of fully methylated $(\mathrm{mC} / \mathrm{mC})$, hemimethylated $(\mathrm{C} / \mathrm{mC}, \mathrm{mC} / \mathrm{C})$, or unmethylated (C/C) CpG positions. (\# indicates biological replica). Note that in bisulfite sequencing, unconverted cytosine $(C)$ must be considered as 5-methylcytosine $(5 \mathrm{mC})$ or 5-hydroxymethylcytosine $(5 \mathrm{hmC})$ and converted $\mathrm{C}$ as unmodified $\mathrm{C}$, 5-formylcytosine (5fC), or 5-carboxycytosine ( $5 \mathrm{caC}$ ), for example $\mathrm{mC}=5 \mathrm{mC}$ / $5 \mathrm{hmC} \mathrm{C}=\mathrm{C} / \mathrm{fC} / 5 \mathrm{caC}$.

\section{Abbreviations}

5mC: 5-methylcytosine; 5hmC: 5-hydroxymethylcytosine; 5fC: 5formylcytosine; 5caC: 5-carboxylcytosine; DHBS: Deep hairpin bisulfite sequencing; dpc: Days post-coitum; ESCs: embryonic stem cells; hpf: Hours post-fertilization; IAP: intracisternal A-particle-LTR1 (IAPLTR1); IF: Immunofluorescence; IVF: In vitro fertilization; L1: Line1Md_Tf; mSat: Major satellites; PGCs: Primordial germ cells; PN: Pronuclear stage; SAMase: S-adenosylmethionine hydrolase. 


\section{Competing interests}

The authors declare that they have no competing interests.

\section{Authors' contributions}

JW and JA conceived the study and wrote the manuscript. JA, MW, KL, and JP designed, performed, and analyzed the experiments and assisted in writing the manuscript. WR supported the research and assisted in writing the manuscript. All authors read and approved the final manuscript.

\section{Acknowledgements}

We thank Jasmin Gries for the sequencing, and Mathias Bader and Pavlo Lutsik for assistance in analyzing the data. This work was supported by a grant from Deutsche Forschungsgemeinschaft (DFG) WA 1029.

\section{Author details}

${ }^{1}$ University of Saarland, FR 8.3, Biological Sciences, Genetics/Epigenetics, Campus A2.4, 66123 Saarbrücken, Germany. ${ }^{2}$ Epigenetics Programme, The Babraham Institute, Cambridge CB22 3AT, UK. ${ }^{3}$ Wellcome Trust Sanger Institute, Hinxton CB10 1SA, UK. ${ }^{4}$ Current address: Departments of Pediatrics and Genetics, Stanford University School of Medicine, 265 Campus Drive, Stanford, CA 94305, USA. ${ }^{5}$ Current address: Departments of Genetics and Obstetrics \& Gynaecology, Stanford University School of Medicine, Institute for Stem Cell Biology \& Regenerative Medicine, 265 Campus Drive, Stanford, CA 94305, USA.

\section{Received: 19 September 2014 Accepted: 26 November 2014}

\section{Published: 9 January 2015}

\section{References}

1. Reik W, Dean W, Walter J. Epigenetic reprogramming in mammalian development. Science. 2001;293:1089-93.

2. Popp C, Dean W, Feng S, Cokus SJ, Andrews S, Pellegrini M, et al. Genomewide erasure of DNA methylation in mouse primordial germ cells is affected by AID deficiency. Nature. 2010;463(7284):1101-5.

3. Smallwood SA, Tomizawa S, Krueger F, Ruf N, Carli N, Segonds-Pichon A, et al. Dynamic CpG island methylation landscape in oocytes and preimplantation embryos. Nat Genet. 2011:43(8):811-4.

4. Guibert S, Forne T, Weber M. Global profiling of DNA methylation erasure in mouse primordial germ cells. Genome Res. 2012;22(4):633-41.

5. Seisenberger S, Andrews S, Krueger F, Arand J, Walter J, Santos F, et al. The dynamics of genome-wide DNA methylation reprogramming in mouse primordial germ cells. Mol Cell. 2012;48(6):849-62.

6. Smith ZD, Chan MM, Mikkelsen TS, Gu H, Gnirke A, Regev A, et al. A unique regulatory phase of DNA methylation in the early mammalian embryo. Nature. 2012;484(7394):339-44.

7. Guo H, Zhu P, Yan L, Li R, Hu B, Lian Y, et al. The DNA methylation landscape of human early embryos. Nature. 2014;511(7511):606-10.

8. Smith ZD, Chan MM, Humm KC, Karnik R, Mekhoubad S, Regev A, et al. DNA methylation dynamics of the human preimplantation embryo. Nature. 2014:511(7511):611-5.

9. Wang L, Zhang J, Duan J, Gao X, Zhu W, Lu X, et al. Programming and inheritance of parental DNA methylomes in mammals. Cell. 2014;157(4):979-91.

10. Morgan HD, Santos F, Green K, Dean W, Reik W. Epigenetic reprogramming in mammals. Hum Mol Genet. 2005;14 Spec No 1:R47-58.

11. Hackett JA, Sengupta R, Zylicz JJ, Murakami K, Lee C, Down TA, et al. Germline DNA demethylation dynamics and imprint erasure through 5-hydroxymethylcytosine. Science. 2013;339(6118):448-52.

12. Yamaguchi S, Hong K, Liu R, Inoue A, Shen L, Zhang K, et al. Dynamics of 5-methylcytosine and 5-hydroxymethylcytosine during germ cell reprogramming. Cell Res. 2013;23(3):329-39.

13. Hajkova P, Jeffries SJ, Lee C, Miller N, Jackson SP, Surani MA. Genome-wide reprogramming in the mouse germ line entails the base excision repair pathway. Science. 2010;329(5987):78-82.

14. Mayer W, Niveleau A, Walter J, Fundele R, Haaf T. Demethylation of the zygotic paternal genome. Nature. 2000;403(6769):501-2.

15. Santos F, Hendrich B, Reik W, Dean W. Dynamic reprogramming of DNA methylation in the early mouse embryo. Dev Biol. 2002:241(1):172-82.

16. Wossidlo M, Arand J, Sebastiano V, Lepikhov K, Boiani M, Reinhardt R, et al. Dynamic link of DNA demethylation, DNA strand breaks and repair in mouse zygotes. EMBO J. 2010;29(11):1877-88.
17. Gu TP, Guo F, Yang H, Wu HP, Xu GF, Liu W, et al. The role of Tet3 DNA dioxygenase in epigenetic reprogramming by oocytes. Nature. 2011;477(7366):606-10.

18. Iqbal K, Jin SG, Pfeifer GP, Szabo PE. Reprogramming of the paternal genome upon fertilization involves genome-wide oxidation of 5-methylcytosine. Proc Natl Acad Sci U S A. 2011;108(9):3642-7.

19. Wossidlo M, Nakamura T, Lepikhov K, Marques CJ, Zakhartchenko V, Boiani $M$, et al. 5-Hydroxymethylcytosine in the mammalian zygote is linked with epigenetic reprogramming. Nat Commun. 2011;2:241.

20. Inoue A, Zhang Y. Replication-dependent loss of 5-hydroxymethylcytosine in mouse preimplantation embryos. Science. 2011;334(6053):194.

21. Arand J, Spieler D, Karius T, Branco MR, Meilinger D, Meissner A, et al. In vivo control of $\mathrm{CpG}$ and non-CpG DNA methylation by DNA methyltransferases. PLoS Genet. 2012;8(6):e1002750.

22. Laird CD, Pleasant ND, Clark AD, Sneeden JL, Hassan KM, Manley NC, et al. Hairpin-bisulfite PCR: assessing epigenetic methylation patterns on complementary strands of individual DNA molecules. Proc Natl Acad Sci U S A. 2004;101(1):204-9.

23. Kim SH, Kang YK, Koo DB, Kang MJ, Moon SJ, Lee KK, et al. Differential DNA methylation reprogramming of various repetitive sequences in mouse preimplantation embryos. Biochem Biophys Res Commun. 2004;324(1):58-63.

24. Lane N, Dean W, Erhardt S, Hajkova P, Surani A, Walter J, et al. Resistance of IAPs to methylation reprogramming may provide a mechanism for epigenetic inheritance in the mouse. Genesis. 2003;35(2):88-93.

25. Oswald J, Engemann S, Lane N, Mayer W, Olek A, Fundele R, et al. Active demethylation of the paternal genome in the mouse zygote. Curr Biol. 2000;10(8):475-8.

26. Studier FW, Movva NR. SAMase gene of bacteriophage T3 is responsible for overcoming host restriction. J Virol. 1976;19(1):136-45.

27. Hajkova P, Erhardt S, Lane N, Haaf T, El-Maarri O, Reik W, et al. Epigenetic reprogramming in mouse primordial germ cells. Mech Dev. 2002;117(1-2):15-23.

28. Sato S, Yoshimizu T, Sato E, Matsui Y. Erasure of methylation imprinting of Igf2r during mouse primordial germ-cell development. Mol Reprod Dev. 2003;65(1):41-50.

29. Habibi E, Brinkman AB, Arand J, Kroeze LI, Kerstens HH, Matarese F, et al. Whole-genome bisulfite sequencing of two distinct interconvertible DNA methylomes of mouse embryonic stem cells. Cell Stem Cell. 2013;13(3):360-9.

30. Shipony Z, Mukamel Z, Cohen NM, Landan G, Chomsky E, Zeliger SR, et al. Dynamic and static maintenance of epigenetic memory in pluripotent and somatic cells. Nature. 2014;513(7516):115-9.

31. Guo F, Li X, Liang D, Li T, Zhu P, Guo H, et al. Active and passive demethylation of male and female pronuclear DNA in the Mammalian zygote. Cell Stem Cell. 2014;15(4):447-58.

32. Shen L, Inoue A, He J, Liu Y, Lu F, Zhang Y. Tet3 and DNA replication mediate demethylation of both the maternal and paternal genomes in mouse zygotes. Cell Stem Cell. 2014;15(4):459-70.

33. Valinluck V, Sowers LC. Endogenous cytosine damage products alter the site selectivity of human DNA maintenance methyltransferase DNMT1. Cancer Res. 2007;67(3):946-50.

34. Nakamura T, Liu YJ, Nakashima H, Umehara H, Inoue K, Matoba S, et al. PGC7 binds histone H3K9me2 to protect against conversion of $5 \mathrm{mC}$ to 5hmC in early embryos. Nature. 2012;486(7403):415-9.

35. Peat JR, Dean W, Clark SJ, Krueger F, Smallwood SA, Ficz G, et al. Genomewide bisulfite sequencing in zygotes identifies demethylation targets and maps the contribution of TET3 oxidation. Cell Rep. 2014; [http://dx.doi.org/ 10.1016/j.celrep.2014.11.034]

36. Booth MJ, Branco MR, Ficz G, Oxley D, Krueger F, Reik W, et al. Quantitative sequencing of 5-methylcytosine and 5-hydroxymethylcytosine at singlebase resolution. Science. 2012;336(6083):934-7.

37. He YF, Li BZ, Li Z, Liu P, Wang Y, Tang Q, et al. Tet-mediated formation of 5-carboxylcytosine and its excision by TDG in mammalian DNA. Science. 2011;333(6047):1303-7.

38. Inoue A, Shen L, Dai Q, He C, Zhang Y. Generation and replicationdependent dilution of $5 \mathrm{fC}$ and $5 \mathrm{caC}$ during mouse preimplantation development. Cell Res. 2011;21(12):1670-6.

39. Kurimoto K, Yabuta Y, Ohinata Y, Shigeta M, Yamanaka K, Saitou M. Complex genome-wide transcription dynamics orchestrated by Blimp1 for the specification of the germ cell lineage in mice. Genes Dev. 2008;22(12):1617-35. 
40. Nagy A, Gertsenstein M, Vintersten K, Behringer R. Manipulating the Mouse Embryo: A Laboratory Manual. 3rd ed. Cold Spring Harbor, NY: Cold Spring Harbor Laboratory Press; 2003. p. 764.

41. Adenot PG, Mercier Y, Renard JP, Thompson EM. Differential H4 acetylation of paternal and maternal chromatin precedes DNA replication and differential transcriptional activity in pronuclei of 1-cell mouse embryos. Development. 1997;124(22):4615-25.

42. Yamagata K, Yamazaki T, Yamashita M, Hara Y, Ogonuki N, Ogura A. Noninvasive visualization of molecular events in the mammalian zygote. Genesis. 2005:43(2):71-9.

43. Yoshimizu T, Sugiyama N, De Felice M, Yeom Yl, Ohbo K, Masuko K, et al. Germline-specific expression of the Oct-4/green fluorescent protein (GFP) transgene in mice. Dev Growth Differ. 1999;41(6):675-84.

doi:10.1186/1756-8935-8-1

Cite this article as: Arand et al:: Selective impairment of methylation maintenance is the major cause of DNA methylation reprogramming in the early embryo. Epigenetics \& Chromatin 2015 8:1.

\section{Submit your next manuscript to BioMed Central and take full advantage of:}

- Convenient online submission

- Thorough peer review

- No space constraints or color figure charges

- Immediate publication on acceptance

- Inclusion in PubMed, CAS, Scopus and Google Scholar

- Research which is freely available for redistribution 